\title{
THE ROLE OF IMAGISTICS INTHE DIAGNOSIS OF CHONDROSARCOMAS
}

doi: 10.2478/rojost-2018-0033

\author{
A.N. Marinescu $U^{1,2}$, A.I. Nicula ${ }^{1,2}$, M.A. Bratu, , G. Iana ${ }^{1,2}$ \\ 1"Carol Davila" University of Medicine and Pharmacy, Bucharest, Romania \\ ${ }^{2}$ Department of Medical Imaging, University Emergency Hospital, Bucharest, Romania \\ ${ }^{3}$ Radiology Department, Colțea Hospital, \\ Bucharest, Romania
}

Chondrosarcomas are malignant cartilaginous tumors, with many histological subtypes and three grades based on cellularity. They are the second most common primary malignant tumors of the bone.

From the histopathologically proved clinical cases, we are reviewing the imaging semiology size, type of calcifications, cortical breach, endosteal scalloping, permeative or moth eaten bone appearance, and revealing other differentiation elements like location, age, and pain. A special point is the frequent difficulty in distinguishing between enchondromas and low grade conventional chondrosarcomas - as the lesions are very similar both histologically and radiographically. The radiologist should point the area to aim on the biopsy - at areas that may harbor foci of high-grade tumor, such as the ones of endosteal scalloping, soft-tissue components, or diffusely enhancing areas with minimal mineralization. It is known that with cartilaginous tumors, histopathologic examination of the biopsy specimen alone does not permit an accurate classification of the tumor. We emphasized the role of imaging in positive and differential diagnosis, management, and therapy of these bone tumors.

Keywords: chondrosarcoma, bone tumors, endosteal scalloping 